\title{
A low psoas muscle volume predicts longer hospitalization and cancer recurrence in upper urinary tract urothelial carcinoma
}

\author{
SOHGO TSUTSUMI $^{1}$, TAKASHI KAWAHARA ${ }^{1,2}$, JUN-ICHI TERANISHI ${ }^{1}$, MASAHIRO YAO $^{2}$ and HIROJI UEMURA $^{1}$ \\ ${ }^{1}$ Department of Urology and Renal Transportation, Yokohama City University Medical Center, Yokohama; ${ }^{2}$ Department of \\ Urology, Yokohama City University, Graduate School of Medicine, Yokohama, Kanagawa 232-0024, Japan
}

Received September 19, 2017; Accepted December 12, 2017

DOI: $10.3892 / \operatorname{mco} .2017 .1537$

\begin{abstract}
The aging population is becoming a primary global problem. The most important alteration that occurs in the body with age, is the loss of skeletal muscle. Previously, sarcopenia, which is associated with the loss of skeletal muscle, has been reported to be associated with the prognosis of cancer and complications. The present study investigated the importance of sarcopenia with regard to the prognosis or postoperative complications of upper urothelial cancer patients who underwent nephro-ureterectomy. A total of sixty patients (male, $n=44$; female, $n=16$ ) underwent nephro-ureterectomy for upper urothelial carcinoma. The psoas muscle volume was calculated at the level of the umbilicus using axial computed tomography images obtained prior to nephro-ureterectomy. The psoas muscle index (PMI) was calculated by the following formula: (right side psoas muscle area at the level of the umbilicus $\left.\mathrm{mm}^{2}\right) /($ body height $\mathrm{m})^{2}$. The median and mean ( \pm standard deviation) ages of the 44 patients were 71 and 68.0 years ( \pm 12.2 years). The lower PMI group demonstrated a significantly poorer recurrence-free survival compared with the higher PMI group (634 vs. 2,317 days, $\mathrm{P}=0.005$ ). In terms of the duration of postoperative admission, the long-admission group ( $\geq 13$ days) demonstrated a significantly lower PMI compared with the short-admission group ( $\leq 12$ days) (383.0 vs. 433.1, $\mathrm{P}=0.039$ ). Although the overall survival of the two groups did not differ significantly, the lower PMI group tended to have a shorter survival period compared with the higher PMI group $(\mathrm{P}=0.080)$. Of the patients with upper urothelial carcinoma, the lower PMI group exhibited a longer postoperative admission period and poorer recurrence-free survival compared with the higher PMI group. The present findings suggest that
\end{abstract}

Correspondence to: Dr Takashi Kawahara, Department of Urology and Renal Transplantation, Yokohama City University Medical Center, 4-57 Urafune-cho, Minami-ku, Yokohama, Kanagawa 232-0024, Japan

E-mail: takashi_tk2001@yahoo.co.jp

Key words: upper urinary tract cancer, nephro-ureterectomy, sarcopenia, psoas muscle sarcopenia is a meaningful factor that should be considered when selecting therapy for upper urothelial carcinoma.

\section{Introduction}

Aging societies are becoming a major global problem (1). The most important change in the body with age is the loss of skeletal muscle. Rosenberg found that the degree of sarcopenia, which represents a loss of skeletal muscle, is correlated with aging. However, there is still no explicit consensus regarding the cut-off point of skeletal muscle loss in patients with sarcopenia (2). The detailed determination and classification of sarcopenia was suggested by the European Working Group on Sarcopenia in Older People (EWGSOP) (1). Regarding the narrow definition of sarcopenia, skeletal muscle loss is the most important factor to consider when determining sarcopenia (2).

We examined the usefulness of skeletal muscle loss as a predictive factor for the length of admission among patients who underwent radical nephro-ureterectomy.

\section{Patients and methods}

Patients. A total of 60 patients (male, $n=44$; female, $n=16$ ) underwent nephro-ureterectomy for upper urothelial carcinoma in Yokohama City University Medical Center (Table I). The institutional review board of Yokohama City University Medical Center (Yokohama, Japan) approved this study, and written consent was obtained from the patients. The patients were followed up every three months for two years and every six months thereafter with cystectomy and computed tomography (CT). The psoas muscle volume was calculated using axial CT images at the level of the umbilicus obtained prior to nephro-ureterectomy. The psoas muscle index (PMI) was calculated by the following formula: (right side psoas muscle area at the level of the umbilicus $\left.\mathrm{mm}^{2}\right) /(\text { body height } \mathrm{m})^{2}$.

Statistical analyses. The patients' characteristics and preoperative factors were analyzed by the Mann-Whitney U test and the chi-squared test. The Kaplan-Meier product limit estimator was used to estimate the progression-free survival (PFS) and overall survival (OS). The duration of the survival was defined as the time between radical cystectomy and tumor progression or death. The log-rank test was performed for the statistical 
Table I. Baseline patient characteristics.

\begin{tabular}{|c|c|}
\hline Variables & $\begin{array}{c}\text { Number }(\%) \\
\text { or (mean, median) }\end{array}$ \\
\hline Number of patients & $44(100.0)$ \\
\hline Age (mean, median) & $68.0,71$ \\
\hline \multicolumn{2}{|l|}{ Side } \\
\hline Left & $33(75)$ \\
\hline Right & $11(25)$ \\
\hline \multicolumn{2}{|l|}{ T stage } \\
\hline is & $3(6.8)$ \\
\hline $\mathrm{a}$ & $6(13.6)$ \\
\hline 1 & $14(31.8)$ \\
\hline 2 & $5(11.4)$ \\
\hline 3 & $16(36.4)$ \\
\hline Unknown & 0 \\
\hline \multicolumn{2}{|c|}{ Pathological grade (low vs. high) } \\
\hline Low & $13(29.5)$ \\
\hline High & $22(50)$ \\
\hline Unknown & $9(20.5)$ \\
\hline \multicolumn{2}{|c|}{ Pathological grade (G1, G2, G3) } \\
\hline Grade1 & $8(18.2)$ \\
\hline Grade2 & $20(45.5)$ \\
\hline Grade3 & $15(34.1)$ \\
\hline Unknown & $1(2.3)$ \\
\hline Recurrence & $24(54.5)$ \\
\hline
\end{tabular}

comparisons. P-values of $<0.05$ were considered to indicate statistical significance.

\section{Results}

The median and mean [ \pm standard deviation (SD)] ages of the 44 patients were 71 and 68.0 years ( \pm 12.2 years), respectively. The median and mean $( \pm \mathrm{SD})$ post-operative hospitalization periods were 12 and 14.5 days ( \pm 6.5 days), respectively. The median and mean $( \pm \mathrm{SD})$ follow-up periods were 1,086 and $1,121.0$ days ( \pm 768.6 days), respectively. The clinicopathological data are summarized in Table I. The survival duration was defined as the time between nephro-ureterectomy and tumor recurrence or death. The median and mean $( \pm$ SD) areas of the psoas at the level of the umbilicus were 1,022.5 and $1,010.1 \mathrm{~mm}^{2}( \pm 302.9)$. The median and mean PMI were 397.1 and $411.6( \pm 98.4)$, respectively.

We divided the patients into two groups based on their PMI (with even numbers of patients). In terms of the duration of postoperative admission, the long-admission group ( $\geq 13$ days) showed significantly lower PMI than the short-admission group ( $\leq 12$ days) (383.0 vs. 433.1, $\mathrm{P}=0.039$ ) (Fig. 1 ). The lower PMI group showed a significantly poorer recurrence-free survival than the higher PMI group (634 vs. 2,317 days, $\mathrm{P}=0.005$ ) (Fig. 2). There were no significant differences in the overall survival between the two groups; however, the lower PMI group tended to have a shorter survival than the higher PMI group ( $\mathrm{P}=0.080)$ (Fig. 3).

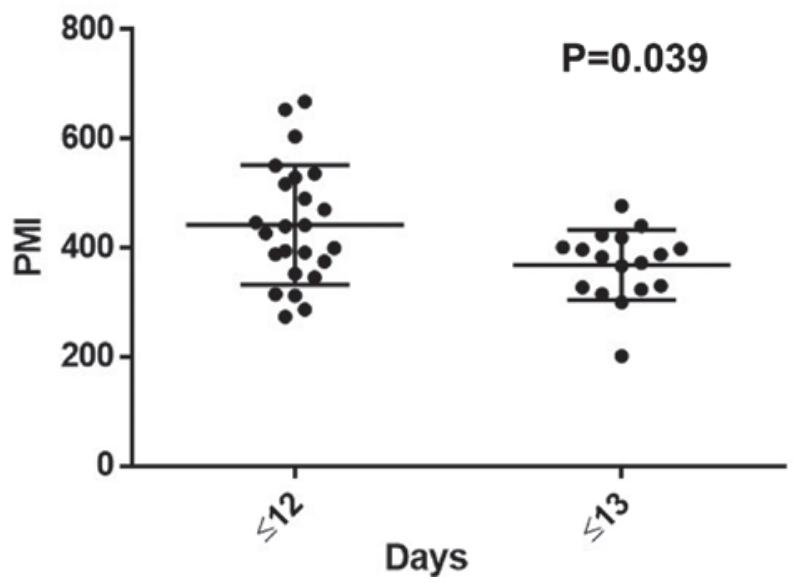

Figure 1. The longer postoperative admission group showed significantly lower PMI values. PMI, psoas muscle index.

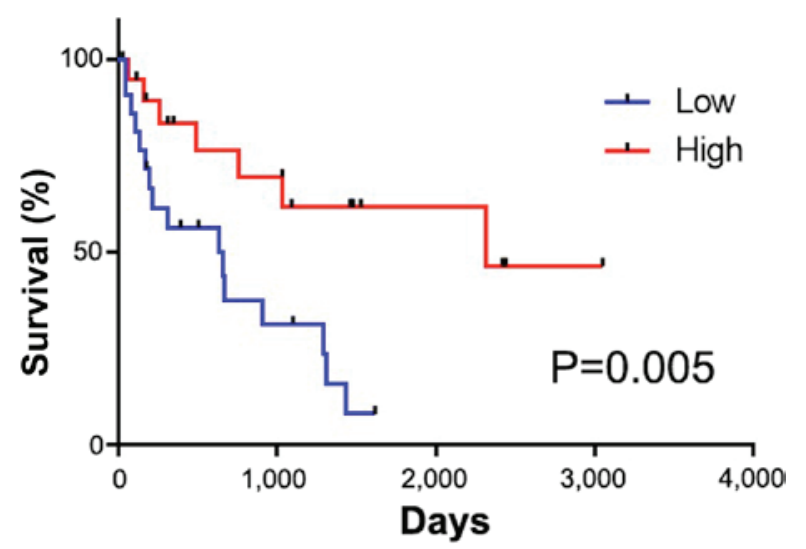

Figure 2. The recurrence-free survival.

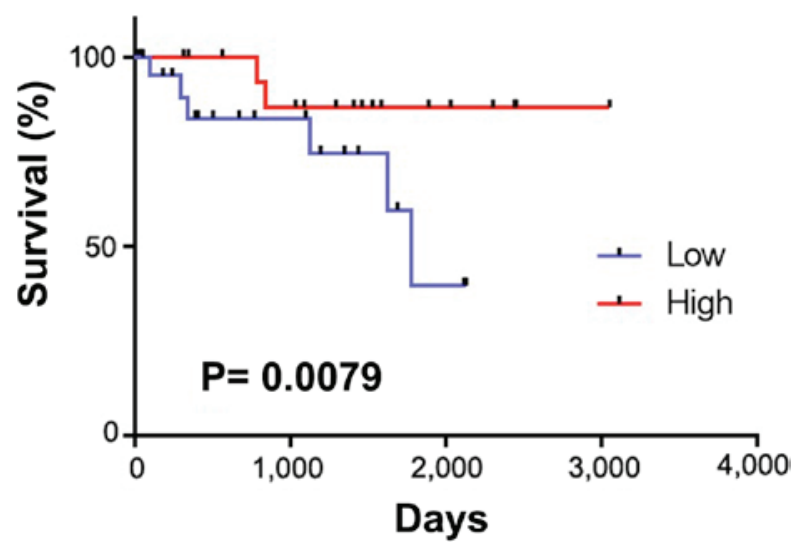

Figure 3. The overall survival.

\section{Discussion}

Upper urothelial carcinoma accounts for $\sim 5 \%$ of all cases of urothelial carcinoma (3). The likely incidence is higher among patients who are 70-80 years of age; $60 \%$ of patients have invasion at the time of the diagnosis. The bladder is the most common site of recurrence, with a recurrence rate of $30-50 \%$ (4-6). 
Recently, sarcopenia, which refers to the loss of skeletal mass that occurs with aging, was found to be a prognostic risk factor for the survival or postoperative complications. Patients with sarcopenia show a worse swallowing ability and a lower nutritional status than those without it. Furthermore, older men $(>65$ years of age) with sarcopenia showed lower ADL values than patients without sarcopenia $(7,8)$.

We previously reported on the impact of sarcopenia using PMI in bladder cancer patients who received systemic chemotherapy and total cystectomy. There as well, a low PMI was associated with a poorer oncological outcome than a high PMI $(9,10)$. Other recent studies have revealed that sarcopenia is a risk factor for postoperative complications or a poor survival in some solid cancers. For stage 2-3 gastric cancer, patients with sarcopenia showed a higher rate of postoperative complications and a poorer overall and recurrence-free survival than those without sarcopenia $(8,11)$. Pancreatic cancer patients with sarcopenia who underwent pancreatectomy also showed a poorer overall survival than the patients without sarcopenia (12).

Although the detailed mechanisms underlying the association between sarcopenia and postoperative complications are still not fully understood, physical weakness reduces the persistence of long admissions of due to the adverse health effects of invasive surgery $(10,13)$. Furthermore, sarcopenia is believed to develop due to aging or malignancy.

In the present study, upper urothelial carcinoma patients with longer admission durations showed lower PMI values than those with shorter admission durations. Previous studies reported a postoperative complication rate of $20.8 \%$ and a postoperative mortality rate at 90 days after surgery of $4.4 \%$ in similar populations $(14,15)$.

Although several treatment options are available for upper urothelial cancer, including nephron-ureterectomy, conservative surgery, ureteroscopy, segmental resection, percutaneous access, and the use of adjuvant topical agents, an increased incidence of advanced-stage cases means that most patients will require nephro-ureterectomy (4). Given that patients with upper urothelial carcinoma tend to be older, surgeons should carefully evaluate the risk of surgery. Due to the small number of patients in the present study, further investigations will be needed to confirm these findings.

In the present study, we showed that sarcopenia predicts a longer hospitalization period and that sarcopenic patients have a significantly worse prognosis than patients without sarcopenia. The present findings suggest that sarcopenia is a meaningful factor that should be considered when choosing therapy for upper urothelial carcinoma.

\section{Acknowledgements}

We would like to thank Dr R. Shimizu, Dr Y. Nakamkura and Dr T. Yamaki for their technical assistance. The present study was supported by grants from KAKENHI grants (16K20152) and from the Ministry of Education, Culture, Sports, Science and Technology of Japan.

\section{References}

1. Cruz-Jentoft AJ, Baeyens JP, Bauer JM, Boirie Y, Cederholm T, Landi F, Martin FC, Michel JP, Rolland Y, Schneider SM, et al; European Working Group on Sarcopenia in Older People: Sarcopenia: European consensus on definition and diagnosis: Report of the European Working Group on Sarcopenia in older people. Age Ageing 39: 412-423, 2010.

2. Rosenberg IH: Sarcopenia: Origins and clinical relevance. J Nutr 127 (Suppl 5): 990S-991S, 1997.

3. Raman JD, Messer J, Sielatycki JA and Hollenbeak CS: Incidence and survival of patients with carcinoma of the ureter and renal pelvis in the USA, 1973-2005. BJU Int 107: 1059-1064, 2011.

4. Rouprêt M, Zigeuner R, Palou J, Boehle A, Kaasinen E, Sylvester R, Babjuk M and Oosterlinck W; European Association of Urology Guideline Group for urothelial cell carcinoma of the upper urinary tract: European guidelines for the diagnosis and management of upper urinary tract urothelial cell carcinomas: 2011 update. European Association of Urology Guideline Group for urothelial cell carcinoma of the upper urinary tract. Actas Urol Esp 36: 2-14, 2012 (In Spanish).

5. Oya M and Kikuchi E; committee for establishment of clinical practice guideline for management of upper tract urothelial carcinoma; japanese urological association: Evidenced-based clinical practice guideline for upper tract urothelial carcinoma (summary-Japanese Urological Association, 2014 edition). Int J Urol 22: 3-13, 2015.

6. Hara T, Fujimoto H, Sakura M, Inokuchi J, Nishiyama H, Miyazaki J, Ohyama C, Koie T, Kikuchi E and Hinotsu S; Cancer Registration Committee of the Japanese Urological Association: Prognostic factors of recurrent disease in upper urinary tract urothelial cancer after radical nephroureterectomy: Subanalysis of the multi-institutional national database of the Japanese Urological Association. Int J Urol 22: 1013-1020, 2015.

7. Shiozu H, Higashijima M and Koga T: Association of sarcopenia with swallowing problems, related to nutrition and activities of daily living of elderly individuals. J Phys Ther Sci 27: 393-396, 2015.

8. Tanimoto Y, Watanabe M, Sun W, Tanimoto K, Shishikura K, Sugiura Y, Kusabiraki T and Kono K: Association of sarcopenia with functional decline in community-dwelling elderly subjects in Japan. Geriatr Gerontol Int 13: 958-963, 2013.

9. Kasahara R, Kawahara T, Ohtake S, Saitoh Y, Tsutsumi S, Teranishi JI, Miyoshi Y, Nakaigawa N, Yao M, Kobayashi K and Uemura H: A Low psoas muscle index before treatment can predict a poorer prognosis in advanced bladder cancer patients who receive gemcitabine and nedaplatin therapy. Biomed Res Int 2017: 7981549, 2017.

10. Saitoh-Maeda Y, Kawahara T, Miyoshi Y, Tsutsumi S, Takamoto D, Shimokihara K, Hayashi Y, Mochizuki T, Ohtaka M, Nakamura M, et al: A low psoas muscle volume correlates with a longer hospitalization after radical cystectomy. BMC Urol 17: 87, 2017.

11. Zhuang CL, Huang DD, Pang WY, Zhou CJ, Wang SL, Lou N, Ma LL, Yu Z and Shen X: Sarcopenia is an independent predictor of severe postoperative complications and long-term survival after radical gastrectomy for gastric cancer: Analysis from a large-scale cohort. Medicine (Baltimore) 95: e3164, 2016.

12. Onesti JK, Wright GP, Kenning SE, Tierney MT, Davis AT, Doherty MG and Chung MH: Sarcopenia and survival in patients undergoing pancreatic resection. Pancreatology 16: 284-289, 2016.

13. Makary MA, Segev DL, Pronovost PJ, Syin D, Bandeen-Roche K, Patel P, Takenaga R, Devgan L, Holzmueller CG, Tian J and Fried LP: Frailty as a predictor of surgical outcomes in older patients. J Am Coll Surg 210: 901-908, 2010.

14. Bastiampillai R, Lavallée LT, Cnossen S, Witiuk K, Mallick R, Fergusson D, Schramm D, Morash C, Cagiannos I and Breau RH: Laparoscopic nephroureterectomy is associated with higher risk of adverse events compared to laparoscopic radical nephrectomy. Can Urol Assoc J 10: 126-131, 2016.

15. Jeldres C, Sun M, Isbarn H, Lughezzani G, Budäus L, Alasker A, Shariat SF, Lattouf JB, Widmer H, Pharand D, et al: A population-based assessment of perioperative mortality after nephroureterectomy for upper-tract urothelial carcinoma. Urology 75: 315-320, 2010. 